\title{
Oral Leukoplakia. A More Challenging Disorder than It Seems ${ }^{\dagger}$
}

\section{Serban Radu Tovaru}

Oral Medicine Discipline, Faculty of Dental Medicine, “Carol Davila” University of Medicine and Pharmacy, 050037 Bucharest, Romania; serban.tovaru@gmail.com; Tel.: +40-072-303-0739

+ Presented at the XV National and III International Congress of the Italian Society of Oral Pathology and Medicine (SIPMO), Bari, Italy, 17-19 October 2019.

Published: 11 December 2019

Treatment of oral leukoplakia remains largely a debatable problem [1]. According to widely accepted opinions the diagnosis of oral leukoplakia should have two steps [2]: the provisory diagnosis should include general patient evaluation (sex, age) and a local thorough oral exam: clinical form, unilocular, multilocular, topographical site. Other diseases that could produce similar lesions should be eliminated following a specific protocol. In the same time, any local favoring factors (smoking, alcohol, other habits, trauma, infections, etc.) should be eliminated. After 3-4 months a definitive diagnosis will be put accordingly [2]. In some instances the lesions could considerably diminish or even disappear (Figures 1 and 2). If the lesions persist a histopathological evaluation with degree of dysplasia, immunological markers and genetic tests are recommended. The degree of dysplasia should be evaluated according to the WHO scale or according to the binary system [3,4]. If possible genetic tests of the surrounding area of the lesion are recommended. Brush cytology is the best method to be used. Following that complex evaluation protocol, the risk of malignisation could be higher or lower.

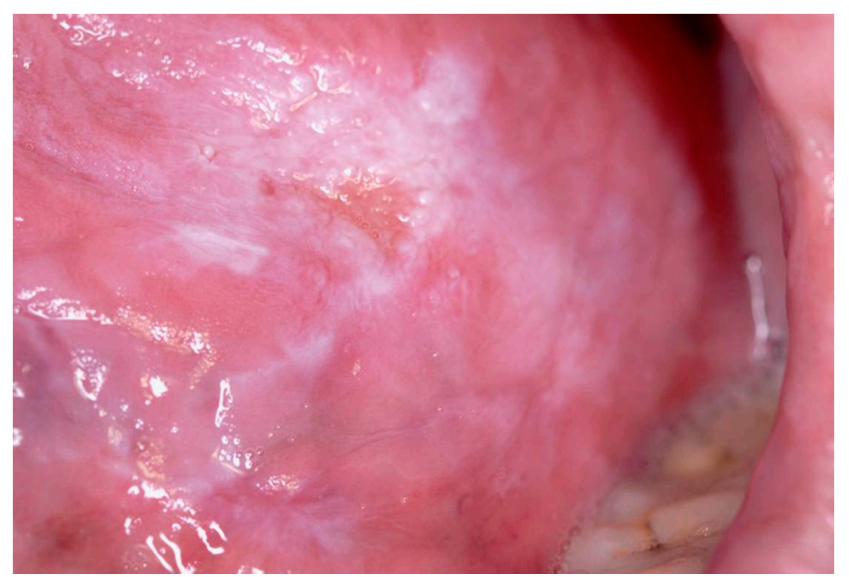

Figure 1. 2015 Male, 70 years, smoker, drinker, Candida positive. Clinical Diagnosis: Non-homogenous diffuse ulcerated lingual leukoplakia. Treatment: Quit smoking, Antifungal treatment. Biopsy. 


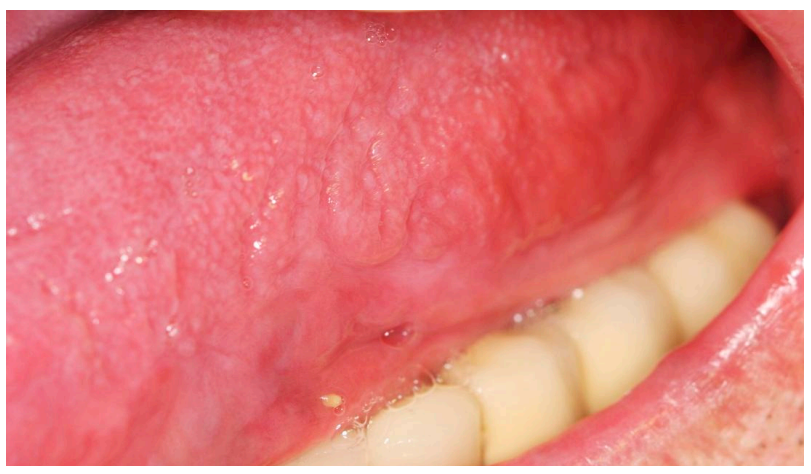

Figure 2. Same patient. 2019. Clinical aspect. The patient quit smoking; 6 months later residual keratotic lesions were removed. No sign of relapse

Lesions with high degree of dysplasia, genetic disturbances (Loss of heterozigosy), multilocular type, speckled, location in high-risk zones should be eliminated. Surgical methods should be adapted according to the width, length, and position of the lesion keeping in mind the borders of oncological security. Classic scalpel removal, laser surgery, cryotherapy should be adapted in accordance with the clinical and histopathological data. A significant percentage of relapse is to be expected in case of idiopathic leukoplakia [1]. A post surgical, strict clinical follow-up must be instituted.

In the same time, some of the patients do not accept a surgical treatment or simply don't come back for control and parts of the lesions evolve without treatment. In this group some of the lesions disappear spontaneously, other enlarge, other shrink and other turn to cancer [5]. Thus, the wait-and-see attitude is an option and a reality. Patients included in this group should be closely followed and if the clinical aspect changes, new biopsies are recommended and a surgical treatment becomes an option. In our opinion that is a valid medical option.

No unified therapeutical guidelines exist so far [1].

Conflicts of Interest: The authors declare no conflict of interest.

\section{References}

1. Holmstrup, P.; Dabelsteen, E. Oral leukoplakia-To treat or not to treat. Oral Dis. 2016, 22, 494-497.

2. Van der Waal, I. Potentially malignant disorders of the oral and oropharyngeal mucosa; terminology, classification and present concepts of management. Oral Oncol. 2009, 45, 317-323.

3. Speight, P.M.; Khurram, S.A.; Kujan, O. Oral potentially malignant disorders: Risk of progression to malignancy. Oral Surg. Oral Med. Oral Pathol. Oral Radiol. 2018, 125, 612-627.

4. Brouns, E.R.A.; Baart, J.A.; Bloemena, E.; Karagozoglu, H.; Van der Waal, I. The relevance of uniform reporting in oral leukoplakia: Definition, certainty factor and staging based on experience with 275 patients. Med. Oral Patol. Oral y Cir. Bucal 2013, 18, e19.

5. Kuribayashi, Y.; Tsushima, F.; Morita, K.I.; Matsumoto, K.; Sakurai, J.; Uesugi, A.; Sato, K.; Oda, S.; Sakamoto, K.; Harada, H. Long-term outcome of non-surgical treatment in patients with oral leukoplakia. Oral Oncol. 2015, 51, 1020-1025. 